\title{
Know Covid-19 and Fight it - An Overview on the Pandemic
}

\section{K Chandrasekharan Nair ${ }^{1 *}$, Pradeep Dathan ${ }^{2}$ and Bheemalingeswara Rao $^{3}$}

${ }^{1}$ Professor Emeritus, Department of Prosthodontics, Sri Sankara Dental College,

Akathumuri, Varkala, Kerala State, India

${ }^{2}$ Professor and Head of the Department of Prosthodontics, Sri Sankara Dental

College, Akathumuri, Varkala, Kerala State, India

${ }^{3}$ Professor, Department of Prosthodontics, Vishnu Dental College, Bhimavaram, AP,

India

*Corresponding Author: K Chandrasekharan Nair, Professor Emeritus, Department of Prosthodontics, Sri Sankara Dental College, Akathumuri, Varkala, Kerala State, India.
Received: May 22, 2021

Published: June 12, 2021

(C) All rights are reserved by $\mathbf{K}$

Chandrasekharan Nair., et al.

\section{Abstract}

Covid-19 pandemic has fallen on our society quite unexpectedly. Luckily the virus responsible for the disease was identified but the vaccines could be introduced after almost an year. By this time, the virus transformed into different strains, further complicating the treatment. Dental practice was affected the most because oral cavity harbours the virus in abundance. This overview provides a compilation of information present for the benefit of practising dentists and dental students.

Keywords: SARS-CoV-2; Disinfection Dental Clinics; Disinfecting Agents; PPE Kit; Precautions for COVID-19

\section{Introduction}

Covid-19 is anxiously as well as curiously observed worldwide by scientists, professionals and common people. The origin of the disease is traced to live animals which were susceptible to coronavirus infection and sold in a food market in Wuhan (China). Animals acted as conduits for the virus to humans. The evolution of the disease was so rapid and has transformed into a public health crisis and spread exponentially throughout the world. At the end of 2019, coronavirus (SARS-CoV-2) with a genome length of 29,903 nucleotides was isolated by a Chinese researcher from a patient with pneumonia. Analysis of the genomic sequence of SARS-CoV-2 showed $88 \%$ identity with two bat-derived SARS-like coronavirus, indicating that mammals acted as the initial reservoir.
The COVID-19 recognised in India is part of the worldwide pandemic. On 30 January 2020, the first case of COVID-19 in India was reported, in Kerala, among three Indian medical students who had returned from Wuhan. India declared a lock down during the last week of March 2020. There was a rapid spread and the first wave of the disease dropped to below 15,000 new cases per day in January 2021. Subsequently a second wave began at a much larger scale than the first in March 2021.

Identifying the future risk, India launched its vaccination programme in January 2021. The British Oxford-AstraZeneca vaccine (Covishield), the Indian BBV152 (Covaxin) vaccine and the Russian Sputnik V vaccine were authorised by the Government of India for 
emergency use. Now India has nearly 20 million reported cases as on May 2021 [1].

\section{What is a 'novel' coronavirus?}

The present pandemic is caused by Novel coronavirus (CoV) which is a new strain of coronavirus. The disease is named coronavirus disease 2019 (COVID-19) - 'CO' stands for corona, 'VI' for virus, and ' $D$ ' for disease (Figure 1). Covid - 19, Severe Acute Respiratory Syndrome (SARS) and some types of common cold are caused by the same family of viruses. Respiratory infections are transmitted through droplets having different sizes. A respiratory droplet gets its designation when the diameter is $>5-10 \mu \mathrm{m}$. When they are $<5 \mu \mathrm{m}$ in diameter, they become droplet nuclei. In airborne transmission, microbes are enclosed in droplet nuclei, the diameter of which is $<5 \mu \mathrm{m}$ and can remain in the air for longer duration. $<5 \mu \mathrm{m}$ particles can travel distances greater than $1 \mathrm{~m}$. A report recently published in The Lancet has stated that 'SARS-CoV-2, the virus that causes COVID-19, is an airborne pathogen'. The report has challenged the popular belief that coronavirus spreads only through smaller aerosols that remain suspended in the air or through fomites [2,3].

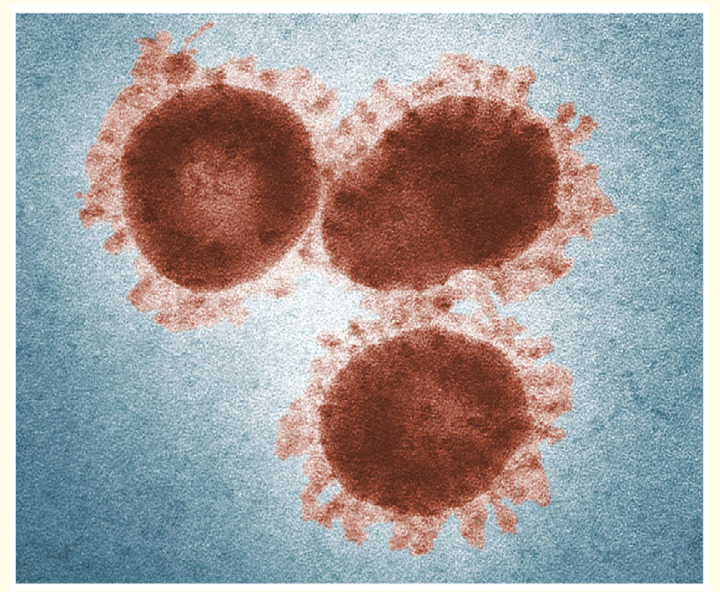

Figure 1: Corona virus - image from transmission electron microscope. Source: https://www.eurekalert.org/pub_ releases/2020-01/c-ub-rdf011620.php.

\section{Symptoms}

People with COVID-19 manifest a wide range of symptoms which may appear in 2 - 14 days after exposure to the virus. People with these symptoms may have COVID-19:
- Fever or chills

- Headache

- Muscle or body aches

- Sore throat

- Cough

- Shortness of breath or difficulty breathing

- $\quad$ Fatigue

- Loss of taste or smell

- Congestion or runny nose

- Nausea or vomiting

- Diarrhoea.

Senior citizens and people who suffer from serious medical conditions like heart or lung disease or diabetes seem to be on the higher side of risk because they may develop serious complications from COVID-19 illness [4].

General procedures to be carried out to avoid the risk of infection

- Wash hands often with soap and water for at least 20 seconds or use an alcohol-based hand sanitizer that contains at least $60 \%$ alcohol as a substitute.

- Avoid touching eyes, nose, and mouth with unwashed hands.

- Avoid close contact with people who are sick.

- Cover the mouth while coughing or sneezing with a tissue and then destroy it.

- Clean and disinfect frequently touched objects and surfaces.

- Maintain at least 1 meter (3 feet) body distance between people, particularly those who are coughing, sneezing and have a fever.

- Wear masks that completely cover the nose and mouth, fit snugly against the sides of face and don't have gaps and have a nose wire to prevent air from leaking out of the top of the mask (Figure 2 and 3).

\section{Common tests used for detecting Covid-19 infection}

\section{RT-PCR test}

The RT in the RT-PCR test stands for reverse transcription and it is an important step in the testing process because SARS-CoV-2 Virus is an RNA based virus. To detect presence of COVID-19 virus, 


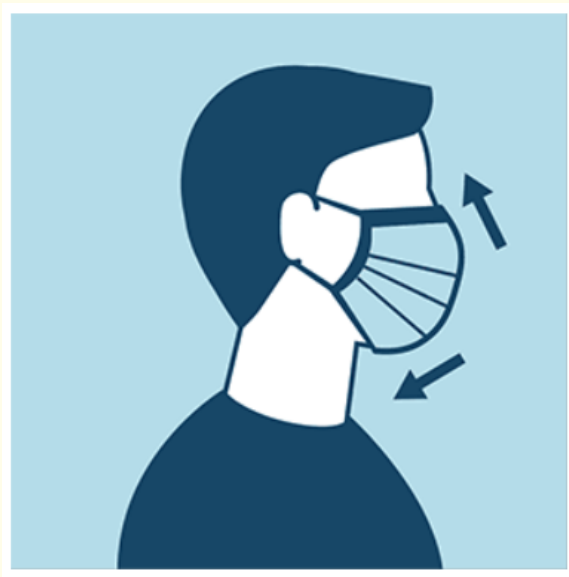

Figure 2: Right way of wearing mask. Source: https://news.llu. edu/health-wellness/which-type-of-face-mask-most-effectiveagainst-covid-19.

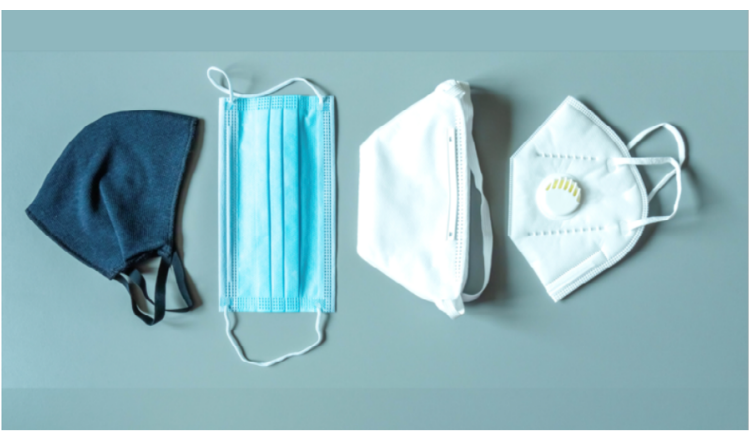

Figure 3: Different types of masks. Source: https://news.llu. edu/health-wellness/which-type-of-face-mask-most-effectiveagainst-covid-19.

scientists have to convert the RNA to DNA. This process is called 'reverse transcription'. The Reverse Transcription of RNA to DNA is carried out because under laboratory conditions, only DNA can be copied or amplified, which is important for the detection of COVID-19 virus. As the number of new copies of viral DNA strands gathers in the sample, marker labels get attached to the DNA strands and then release a fluorescent dye. The computer of the testing ma- chine tracks the quantity of fluorescence in the sample after each cycle and once a certain threshold is surpassed, the presence of the virus is confirmed.

\section{Antigen test}

Antigen test and RT-PCR use almost the same method - that is using nasal fluid in a swab. Antigen test is considered as a rapid test in which the sample is mixed with a solution that unleashes specific viral proteins. The combined solution is then applied to a paper strip containing optimised antibody which binds with these proteins if they are present. The result is obtained as a band on the paper strip. The test doesn't need a laboratory and can be completed in 30 minutes, but the speed very often limits the sensitivity. Although these tests are reliable when an individual has a high viral load. Antigen tests are prone to false-negative results if the virus in the body is less. However, it is not as expensive as RTPCR test.

\section{Rapid antibody test}

Rapid Antibody Test looks for antibodies present in a patient's blood samples. According to ICMR,' these tests are mainly used for mass analysis and reporting'. Antibody testing determines whether a person had suffered from COVID-19 in the past and at present has antibodies against the virus. Antibody testing is also known as serology testing. A health care professional takes a blood sample, usually by a finger prick or by drawing blood from a vein in the arm. Then the sample is tested to determine whether one has developed antibodies against the virus. The immune system produces these antibodies (proteins) that are critical for fighting and clearing out the virus [5].

\section{Disease transmission and dental profession}

Dental profession has a high potential to transmit Covid - 19 because of the following reasons:

1. Oral mucous membrane has a high affinity for Angiotensin converting enzymes receptor 2 (ACE2) which is responsible for the entrance of the virus into human cells and subsequent replications of the virus. That is reason for the increased presence of virus in saliva.

SARS-CoV-2 virus has been detected in saliva samples of 87 - $100 \%$ of clinical patients. In a study on COVID-19 positive patients, it was shown that posterior oropharyngeal saliva samples were serially positive for viral load from the onset of 
symptoms up to 25 days. However, the serum samples were positive only for a fortnight. For the presence of SARS-CoV-2 in the saliva, three pathways have been identified: (i) the direct exchange of secretions from the upper and lower respiratory tract to the oral cavity (ii) gingival crevicular fluid is rich in blood components and can also add viruses to the saliva (iii) major and minor salivary glands can be a source of the virus because epithelial cells of salivary gland ducts are found to be an early target for SARS-CoV infections through ACE2 receptors [6].

2. While undertaking different clinical steps, aerosols are generated. All the dental procedures, performed with highspeed rotating handpiece, ultrasonic scaler and water air syringe are termed as aerosol generating procedures (AGPs). Aerosols are mostly contaminated with blood, bacteria, viruses and fungi.

3. Aerosol refers to liquid and solid particles $(\leq 5 \mu \mathrm{m})$ which dehydrate and remain in the air. It is assumed that aerosols can persist in the air for a very long time and can spread completely in enclosed spaces. Aerosols are estimated to travel between 4 to $8 \mathrm{~m}$ (Figure 4 ).

4. Close proximity of the operator to the patient who can generate aerosols while talking, breathing, coughing or sneezing.

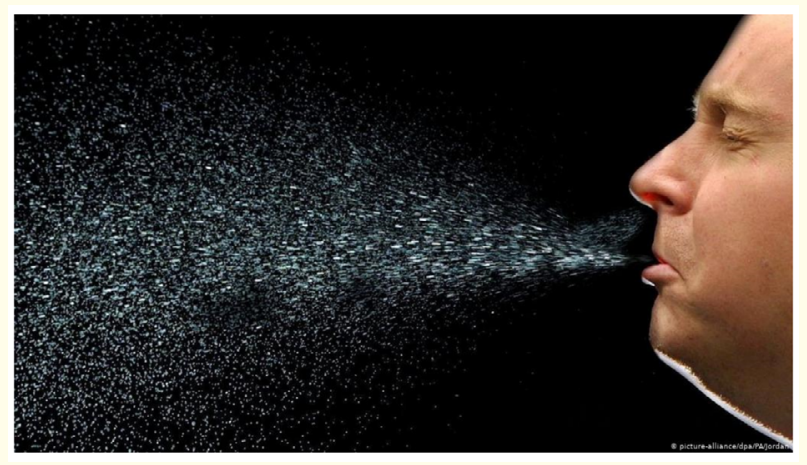

Figure 4: Aerosol. Source: https://www.dw.com/en/dangersof-covid-19-aerosols-are-underestimated/a-54115303.

\section{Preventive care in dental clinics}

While providing dental care standard precautions should be implemented which include health clearance, vaccination for health- care workers, hand hygiene, personal protective equipment (PPE), instrument decontamination, safe sharps handling and sharps injury management, environmental cleaning and disinfection and clinical waste management.

Standard PPE consist of a surgical mask, face shield (usually with inferior opening) or protection goggles, gown, surgical cap, and gloves. Full or enhanced PPE include a surgical mask, face shield or protection goggles, disposable long-sleeved gown (waterproof), surgical cap and gloves in addition to respirators such as N95/FFP2 (filtering face pieces), respirators or powered air purifying respirators. FFP1 refers to the least filtering with an aerosol filtration of at least $80 \%$ for $0.3 \mu \mathrm{m}$ particles and is mainly used as an environmental dust mask. FFP2 masks have a minimum of $94 \%$ filtration percentage and FFP3 masks have filtration percentage of $99 \%$. N95 masks are so called because they are able to filter at least $95 \%$ of very small particles, including droplets containing the coronavirus (Figure 5 and 6). Sequence of putting on PPE is - wash hands - gown - mask - face shield - gloves. Sequence of removing PPE is - gloves - face shield - mask - gown - wash hands (Figure 7 and 8).

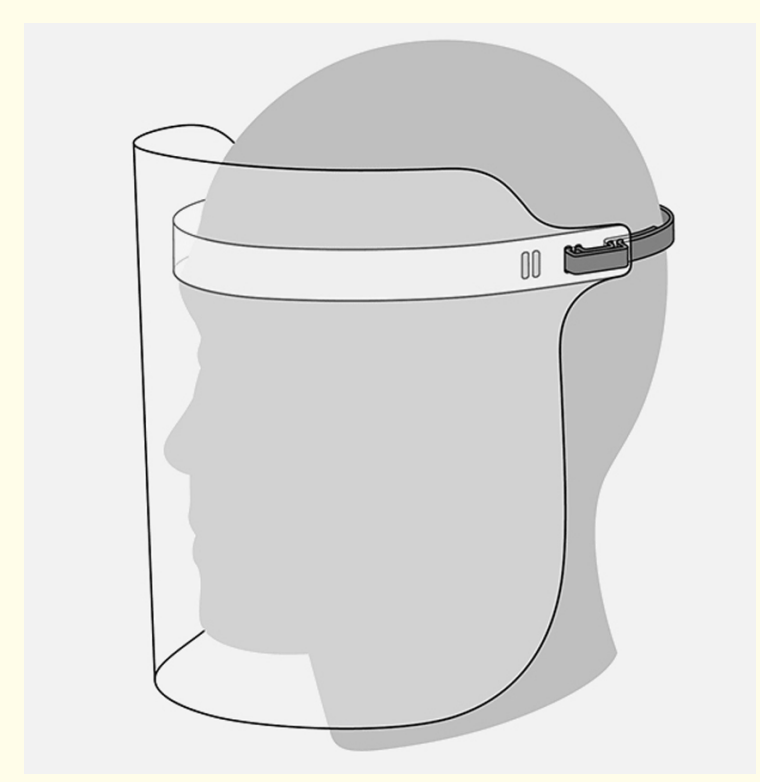

Figure 5: Face shield. Source: https://www.dezeen. com/2020/04/09/apple-coronavirus-face-shield/. 
Masks are generally loose fitting and covers the nose and mouth.

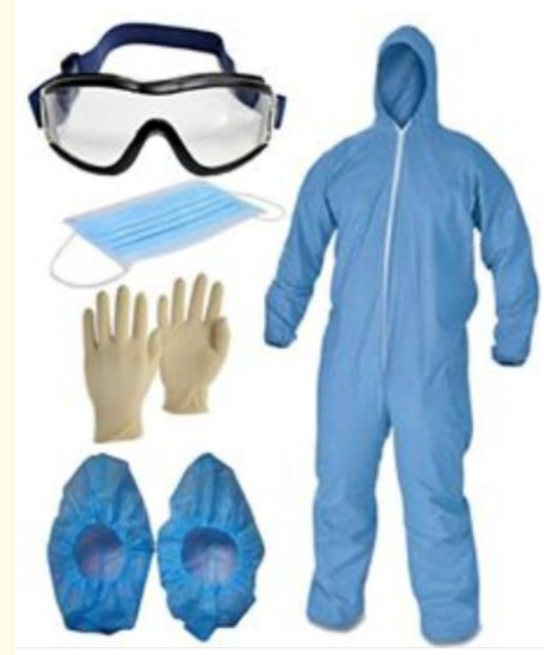

Figure 6: PPE kit. Source: https://www.indiamart.com/ proddetail/ppe-kit-22252032912.html.

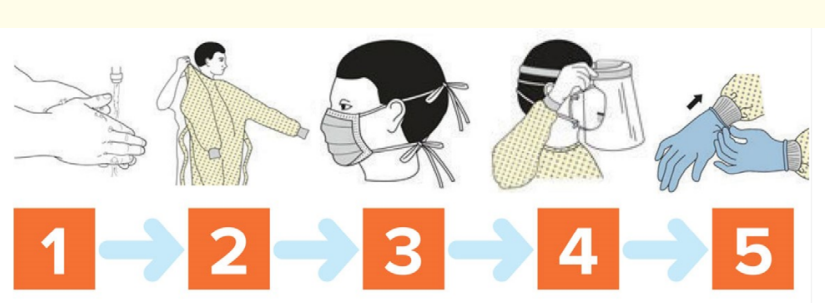

Figure 7: Sequence of putting on PPE.

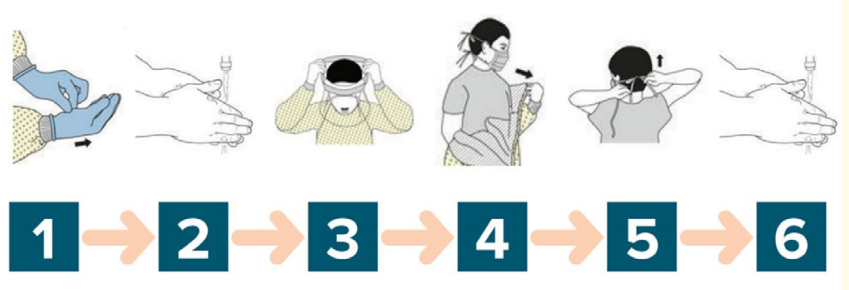

Figure 8: Sequence of removing PPE. Source: https://www. dentalcare.com/en-us/professional-education/ce-courses/ ce483/putting-on-and-safely-removing-ppe.
They are designed for one way protection, to capture bodily fluid leaving the wearer. Whereas respirators are tight fitting masks, designed to create a facial seal. Respirators (non valve) provide good two-way protection, by filtering both inflow and outflow of air.

During surgical procedures, it is recommended to apply preprocedural mouth rinses with chlorhexidine gluconate (CHX), povidone-iodine (PVP-I) or hydrogen peroxide $\left(\mathrm{H}_{2} \mathrm{O}_{2}\right)$ and to put the patient in a supine position to avoid working in the breath way of the patient. Contaminated aerosols are filtered either by chairside high-volume evacuators (HVE) or more expensive HEPA (high efficiency particulate arrestor) filters. HVE filters reduce contamination by around $90 \%$ while HEPA filters can remove $99.7 \%$ of particles measuring $0.3 \mu \mathrm{m}$ in diameter.

The possibility of Covid 19 transmission in dental clinics must be recognised and controlled efficiently, while dealing with patients with suspected or confirmed COVID-19 in order to prevent spreading primarily amongst health care workers. Preventive measures will also avoid cross-infection between patients especially in out-patient settings with poor ventilation and air filtering [7].

Dental care providers are advised to:

1. Follow standard hand hygiene practice, considering the fact that people touch their face on an average of 23 times per hour, with $44 \%$ of these involve the mucous membranes of the mouth and or nose [8].

2. It is preferred to take extraoral radiographs like panoramic radiographs to avoid gag reflex and possible droplet production.

3. Limit the number of staff when the clinical procedures are undertaken to only those essential for patient care and procedure support.

4. Use a dental hand-piece with anti-retraction function. Highspeed dental handpieces without anti-retraction valves may aspirate and expel debris and fluids into the water lines during the dental procedures. More importantly, the microbes, including bacteria and virus, may further contaminate the air and water tubes within the dental unit, and thus can potentially cause cross-infection. Draw back or suck back is a phenomenon seen with airotor handpiece. When the drive 
air stops, the turbine continues to spin. This creates negative pressure in the system and begins to draw aerosols back into the system. Each time the airotor is activated, the drawback, forces the contaminants further into the handpiece, past the coupling and eventually into the air lines. Airline cannot be disinfected. Manufacturers have started including a braking system to reduce the spinning motion of the turbine once the drive air stops.

5. Minimize the use of ultrasonic instruments and 3-way syringes.

6. Resorbable sutures which last 3 to 5 days in the oral cavity should be used to eliminate the need for a follow up appointment.

7. To disinfect different surfaces of the clinic, many disinfectants are used alone or in combinations in healthcare settings. These include alcohols, chlorine compounds, formaldehyde, glutaraldehyde, hydrogen peroxide, iodophors, peracetic acid, phenolics, and quaternary ammonium compounds [9].

\section{Disinfection of dental clinic}

Common sterilization protocols adopted regularly in the dental clinics are effective for the prevention of COVID-19 cross-infection. Commonly used autoclaves are effective because the virus cannot survive more than 30 minutes at temperatures above $56^{\circ} \mathrm{C}$. Sanitizing must be carried out on all surfaces of the dental unit, spittoon area and dentist and assistant's stool. A spray of aqueous solution of hypochlorite at $1 \%$ or alcohol at $70 \%$ can be used and it can be left for at least 1 minute. Wipe the sprayed area with disposable clothes taking care not to go back to previously wiped areas. Water lines of the dental unit can be cleaned with a $0.5 \%$ hypochlorite solution, because residual water may be contaminated by viruses and bacteria. It is obligatory to carry out complete air change of the clinical space after each intervention because SARS-CoV-2 is able to remain suspended in aerosol for up to 3 hours. Continuous air exchange is possible through the use of air suction, filtration, UV lights, ozone generators and portable air cleaners with HighEfficiency Particulate Air (HEPA) filters. Windows with at least 2 square meter openings ensure efficient air exchange in $10 \mathrm{~min}$ for a 20 square meter room; they must be opened after each patient $[10,11]$ (Figure 9).
Figure 9: Medical grade HEPA filters. Source: https://www. amazon.in/Medify-Medical-Filtration-Air-Purifier/dp/ B08194ZQ4N.

\section{Dentists' response}

In a survey conducted by Rodrigues L., et al. dentists have made the following observations:

1. Majority of dentists believed that they were at higher risk of contracting the virus than any other healthcare professionals. They also felt that dentists can transmit the disease too.

2. Dentists believed that wearing PPE did not guarantee them safety against the virus and practicing amidst COVID-19 was not safe.

3. As a career, the pandemic has affected the prospects of dentistry but still it remains to be a lucrative branch.

4. Covid has affected the revenue and the patient inflow is also reduced [12].

\section{Conclusion}

Due to the rapidly evolving nature of Covid-19 virus, it is germane for all oral health professionals to update themselves with new information. Precautionary measures are highly recommended until we understand the specific behaviour and infectious nature of the novel SARS-CoV-2 virus is understood in the light of the evidence collected from research which may eventually lead to the development of more effective vaccines and medications. 
Though the pandemic has pushed both the professionals and public into a stressful situation, the profession has stood up to show grit in the fight against Covid 19. Many dentists have expanded their role beyond dentistry by offering voluntary service by virtue of their training and practical experience. Providing emergency service itself is a great opportunity to prove the relevance of the profession and no doubt we will pull through this crisis and emerge stronger than before.

\section{Bibliography}

1. https://en.wikipedia.org/wiki/COVID-19_pandemic_in_India\#

2. https://www.who.int/news-room/commentaries/detail/ modes-of-transmission-of-virus-causing-covid-19

3. Trisha Greenhalgh, Ten scientific reasons in support of airborne transmission of SARS-CoV-2". The Lancet 397 (2021): 1603-1604.

4. https://www.cdc.gov/coronavirus/2019-ncov/symptomstesting/symptoms.html

5. https://www.medicaldevice-network.com/features/typesof-covid-19-test-antibody-pcr-antigen

6. Infection control in dentistry during COVID - 19 pandemic: what has changed? (2020): e05402.

7. Essam Ahmed Al-Moraissi., et al. "Is Standard Personal Protective Equipment Effective Enough To Prevent COVID-19 Transmission During Aerosol Generating Dental, Oral and Maxillofacial Procedures? A Systematic Review (2020).

8. Kwok YL., et al. "Face touching: a frequent habit that has implications for hand hygiene". American Journal of Infection Control 43 (2015): 112-114.

9. https://psnet.ahrq.gov/primer/covid-19-and-dentistry-challenges-and-opportunities-providing-safe-care

10. Siegel JD., et al. "Health Care Infection Control Practices Advisory Committee. Guideline for isolation precautions: Preventing transmission of infectious agents in health care settings". American Journal of Infection Control 35 (2007): S65-164.
11. Behzad Mostoufi., et al. "COVID-19 and the dental profession: Establishing a safe dental practice for the coronavirus era". Journal of Global Oral Health 3.1 (2020): 41-48.

\section{Volume 5 Issue 7 July 2021}

(C) All rights are reserved by K Chandrasekharan Nair., et al. 